\title{
Transmission Power Optimization and Analysis for SMF considering Optical Pulse Broadening for 193.548 THz
}

\author{
Harish V. Rajurkar \\ Dept. of Electronics \& Telecommunication Engineering \\ Shri Sant Gajanan Maharaj College of Engineering, \\ Shegaon-444203, India
}

\begin{abstract}
The transmission of information through optical fiber is affected by various types of dispersions which reduce the efficiency and hence data carrying capacity of a fiber .But the most important kind of dispersion which affects a lot on the performance of the fiber is Polarization Mode Dispersion, PMD. PMD can cause the optical receiver to be unable to interpret the signal correctly, and results in high bit error rates. This paper demonstrates the broadening of pulse caused by polarization mode dispersion (PMD).
\end{abstract}

\section{General Terms}

Optical Fiber, Attenuation in fiber

\section{Keywords}

SMF, Dispersion, PMD, BER

\section{INTRODUCTION}

The subject of Polarization mode dispersion (PMD) presents many challenges, including understanding the inconsistent terminology and the statical nature of PMD. Polarization mode dispersion (PMD) is one of the main limiting factors of high-speed long distance all optical network. PMD can be a serious limitation on certain fiber links operating at $10 \mathrm{~Gb} / \mathrm{s}$. As network data rates rise, it is becoming increasingly important to understand PMD and its potential impact on your network. [8]

\section{IMPORTANCE OF PMD}

A single Mode Fiber (SMF) is designed to support only one mode of propagation of light .The principal advantage of letting the light pulse prorogates along only one mode i.e. intermodal dispersion can be avoided .Because of only one mode of propagation in SMF intermodal dispersion can be avoided. In spite of the measures taken to provide a symmetrical core cross section, there is some asymmetry in the fiber core. [8]

This change in the symmetry of the fiber core is due to the presence of Birefringence. When the light pulse is propagated through the fiber core, it get decomposed in to two orthogonally polarized components that prorogates through the core with different velocities. Due to this the pulse arrived at the destination with some induced delay ,known as Differential Group delay (DGD), because of which the pulse broadening takes place .This effect on the pulse is known as Polarization Mode Dispersion .[1]The average value of DGD is nothing but PMD .For symmetrical Fiber refractive index are different for $\mathrm{x}$ axis and $\mathrm{y}$ axis. This difference in the refractive index is known as Birefringence. The effect of this Birefringence is that ,a delay will introduce in the pulse .[1] From the past century the growth of Telecommunication technologies is tremendously increasing . There is a tremendous increase in the demand of bandwidth for the higher data rates because of internet.To transfer the data with a faster rates the phenomena like PMD must be considered. It is because higher the data rates less is the separation between the pulses and more the chances of ISI because of PMD, pulse broadening. [5]

\section{CAUSES OF PMD}

There are many ways in which a fiber can become birefringent. Birefringence can arise due to an asymmetric fiber core or can be introduced through internal stresses during fiber manufacture or through external stresses during cabling and installation. Optical fiber manufacturing processes are designed to yield fibers with a circular cross-section. Any deviation from this form will generally result in an elliptical core, which in turn will result in a refractive index difference between the $\mathrm{X}$ and $\mathrm{Y}$-axes of the elliptical core. Even if the fiber core is manufactured with an ideal circular cross-section its refractive index can be asymmetric across its cross-section due to stresses built into the fiber during the manufacturing process or stress that is externally applied during deployment or operation. External asymmetric stresses can be introduced to the fiber during cabling and installation. Any non-uniform loading of the fiber cross-section, or bends or twists that are introduced to the fiber by sub-optimal cabling or installation will result in an asymmetric external stress being placed on the fiber. An optical fiber will exhibit birefringence as a consequence of all of the above sources of internal and external stress. [9]

The birefringence in the optical fiber slows down the $\mathrm{X}$ polarized state that sees the higher refractive index and causes a differential group delay (DGD) between the polarization states that results in pulse distortion often referred to as pulse splitting [1]

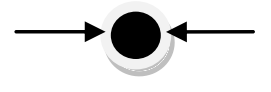

Buit in Stress

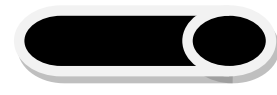

Twist
Fig1 Sources of Birefringence 


\section{THE IMPACT OF PMD}

\subsection{ISI}

Distortion in the pulse that arises when time slot of individual pulse is stretched by PMD induced delay to the point where trail end of leading pulse overlaps with the leading edge of subsequent pulse, this is called ISI. The stretching of pulse reduce the upper and lower level representing 1 and 0 and so introduces system penalty because receiver may not be able to decide between $1 \& 0$. [9]

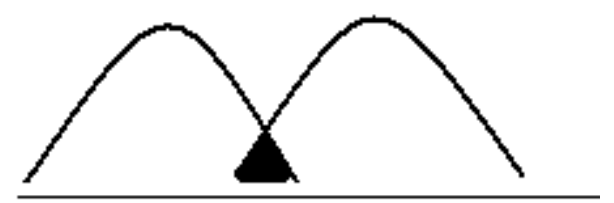

Fig 2 ISI

\subsection{Pulse broadening}

The Single mode fiber segment becomes bi modal due to birefringence induced by these stresses .The propagation constants along the two modes are slightly different .Therefore a DGD will developed in the fiber segment causing pulse broadening. [2]

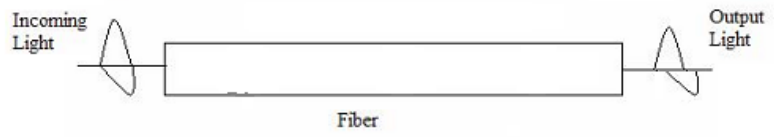

Fig 3 PMD

\subsection{Increase BER}

DGD can disperse the transmitted optical bit and errors at the receiver .If there are relatively few bit errors at the receiver, then usually other mechanisms of the transmission systems can satisfactorily recover the lost transmitted information .However ,if the bit errors are too numerous, then the transmitted information is too corrupt to recover [4].

\section{SIMULATION SETUP}

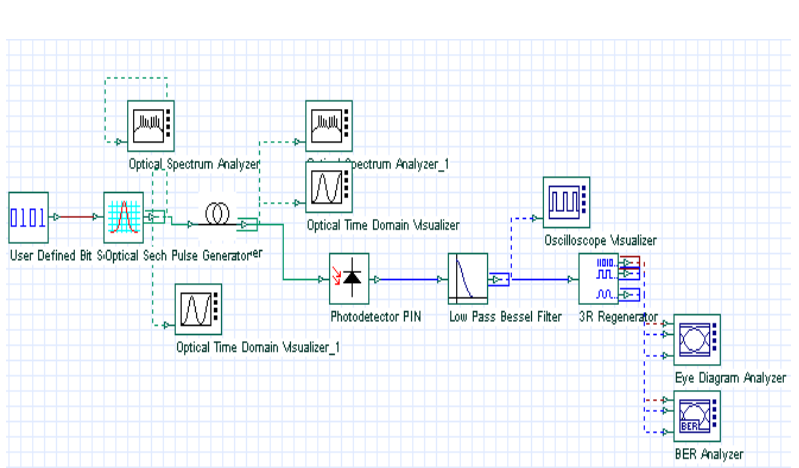

Fig 4 Simulation Set up

In the simulation a data stream 1010 is modulated over with a carrier frequency of $193.548 \mathrm{THz}$. i. e. in the wavelength of $1550 \mathrm{~nm}$ with a power of $3 \mathrm{~W}$.At the receiver section, the optical channel is detected by a PIN detector and analyzes the received electrical signal by connecting Eye Diagram Analyzer and BER analyzer. At the transmitter side the OTDR to analyze the transmitted optical signal is connected. This OTDR display the pattern of transmitted signal and the power associated with it .The same type of OTDR is connected at the receiver end to analyze the optical signal after travelling certain distance. In this set up the performance of the optical pulse is analyzed based on the length of optical fiber cable .Here when the fiber length is varied then how the pulse is dispersed and its effect on the BER are analyzed and also the received power is measured. Here the bit rate is calculated as 40e+009.[6]

\section{RESULT AND DISCUSSION}

\subsection{For Fiber length $L=100 \mathrm{Km}$}

The figure 5, shows the applied bit stream 1010 with a carrier signal of frequency $193.548 \mathrm{THz}$ with a power of about 3 mwatt.This is the data that is to be transfer over the optical fiber cable.

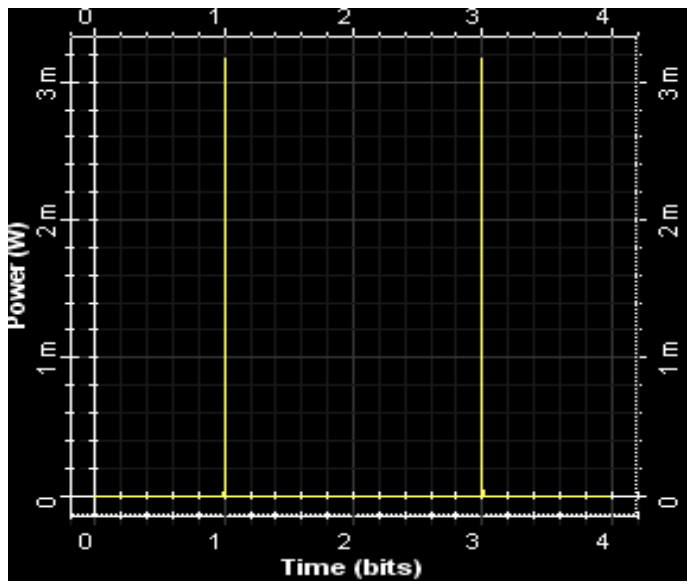

Fig 5. Input bit Stream 1010

Fig 6 shows the nature of data stream when transferred over a fiber cable with a length of $100 \mathrm{Km}$. From fig 8 , it has been found that the data is dispersed i.e. its time slot as compare to the fig 7 is increased .It has been also found that the transmitted power is reduced to around $60 \mu$ watt.So here for a distance of $100 \mathrm{Km}$ power is reduced from 3 mwatt to $60 \mu$ watt.

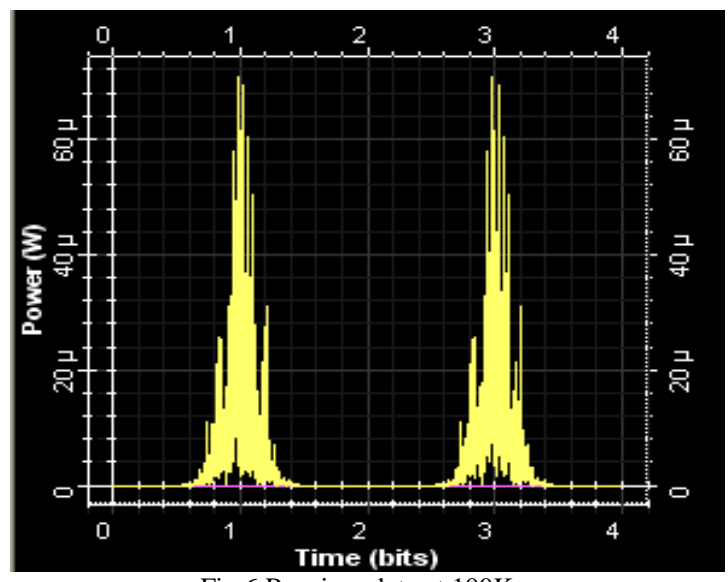

Fig 6.Receiver data at $100 \mathrm{Km}$

Fig 7. Shows the Eye diagram of the received pulse. From the fig the following parameter had been observed.

\begin{tabular}{|l|l|}
\multicolumn{2}{c}{ Table I } \\
\begin{tabular}{|l|l|}
\hline Max Q Factor & $6.56323 \mathrm{e}+044$ \\
\hline Eye Height & $1.31265 \mathrm{e}-005$ \\
\hline
\end{tabular}
\end{tabular}




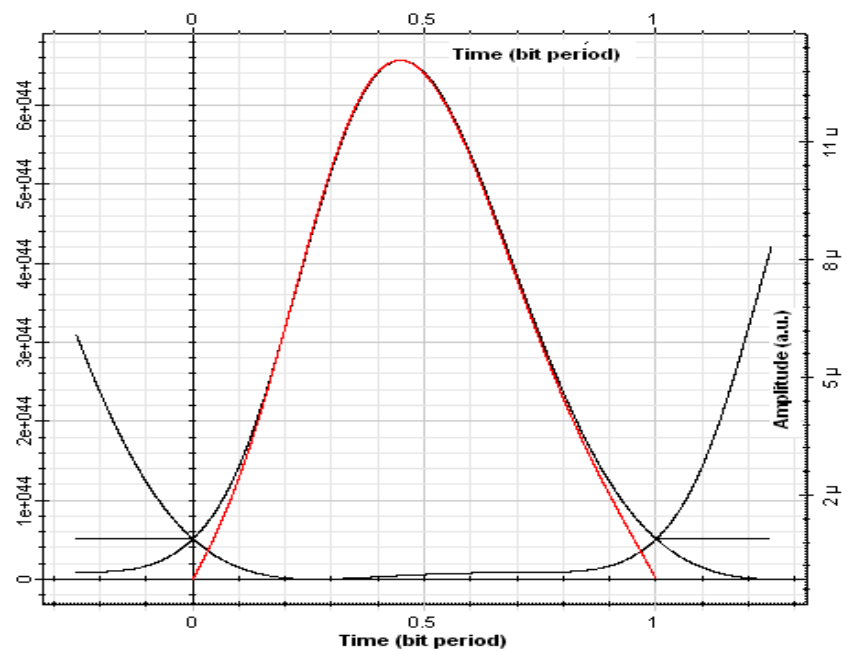

Fig 7. Eye Diagram for Receiver data at $100 \mathrm{Km}$

\subsection{For Fiber length $\mathrm{L}=300 \mathrm{Km}$}
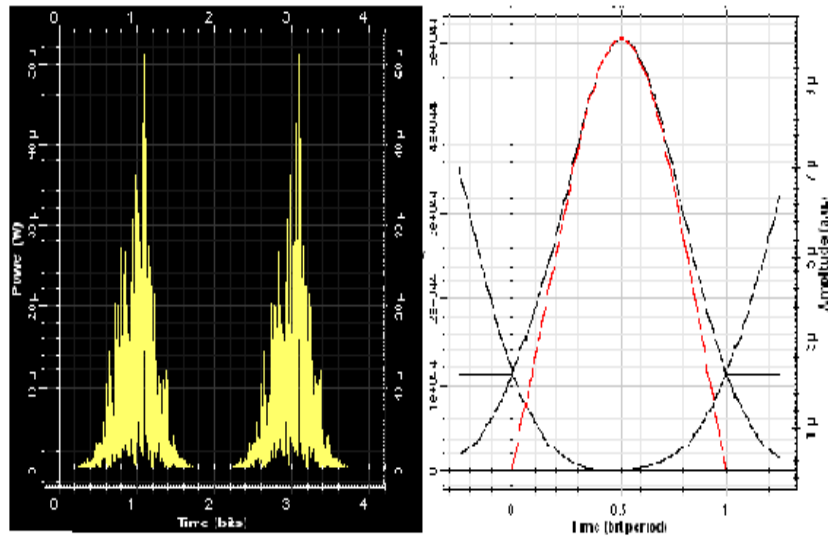

Fig 8. (a,b) Received Data, Eye Diagram for300 Km

From fig $8(a)$ it is observed that when the transmission distance is further increased up to $300 \mathrm{Km}$ the input data stream is disperses further as compare to the fig 3. Also the power which is obtained is $30 \mu \mathrm{W}$.The eye Diagram is shown in the Fig 8(b) from which following parameter had been observed.

Table II

\begin{tabular}{|l|l|}
\hline Max Q Factor & $5.04986 \mathrm{e}+044$ \\
\hline Eye Height & $1.00997 \mathrm{e}-005$ \\
\hline
\end{tabular}

\subsection{For Fiber length $\mathrm{L}=900 \mathrm{Km}$}
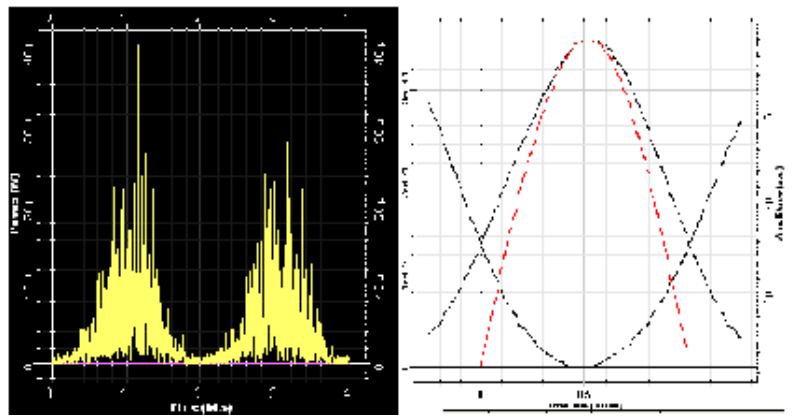

Fig 9 (a.b) Eye Diagram for Receiver data at $900 \mathrm{Km}$
From the fig 9, the following parameter are calculated as, Table III

\begin{tabular}{|l|l|}
\hline Max Q Factor & $3.53467 \mathrm{e}+044$ \\
\hline Eye Height & $7.06933 \mathrm{e}-006$ \\
\hline
\end{tabular}

The power receives after covering a distance of $900 \mathrm{Km}$ is $20 \mu \mathrm{W}$.Here by using the same procedure the all above parameter have been calculated for the transmission distance of $100 \mathrm{Km}, 300 \mathrm{Km}, 900 \mathrm{Km}, 1500 \mathrm{Km}, 2000 \mathrm{Km}$ and the results is given in the following Graphs,

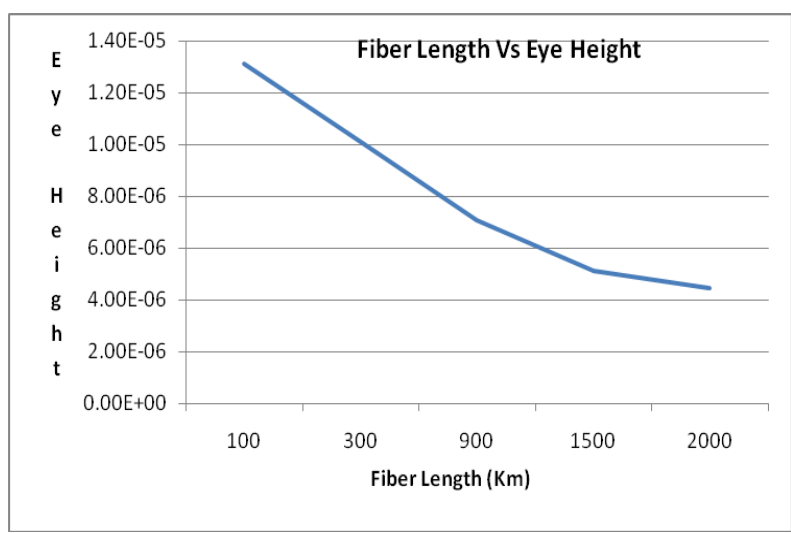

Fig10.Fiber Length (Km) Vs Eye Height

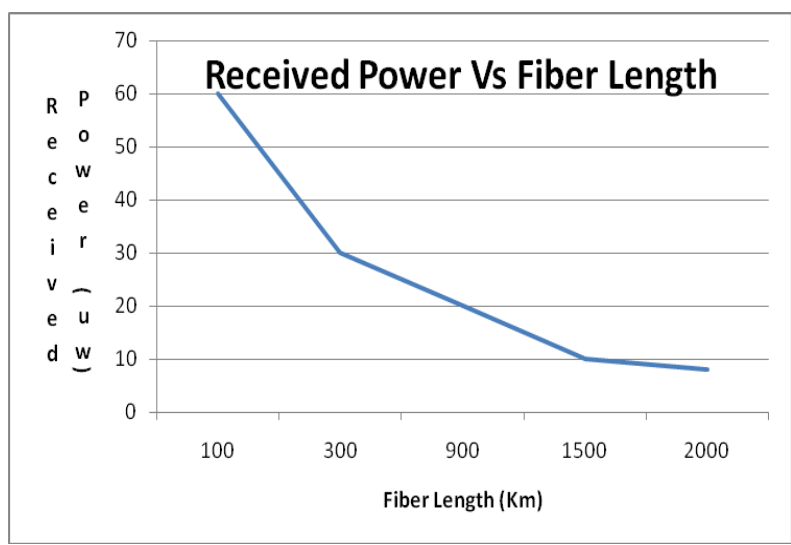

Fig 11: Fiber Length (Km) Vs Rx Power

\section{CONCLUSION}

The effects of change in the transmission distance or Fiber length over the transmitted optical pulse are analyzed. Here the very important issue is the behavior of the pulse as it travels through the fiber channel .Here from the fig 10, it is found that for the fiber of length more than $1500 \mathrm{Km}$ the opening of Eye is less and before $1500 \mathrm{Km}$ of fiber length eye opening is wider which means the dispersion of the pulse is more for fiber of $1500 \mathrm{Km}$ and it is less for the fiber of length less than $1500 \mathrm{Km}$.So, as the pulse propagates through the optical channel ,it get spread depending upon the length of the fiber. So, if this is not taken in to consideration then the may be chances of ISI because of which the reception of the signal may not be proper and hence the signal is not correctly received.

\section{ACKNOWLEDGMENTS}

I am sincerely thankful to the authorities of Shri Sant Gajanan Maharaj College of Engineering, Shegaon for permitting me to use the research facilities in the Photonics Laboratory. 


\section{REFERENCES}

[1] B.W.Hakki,"Polarization mode dispersion in a single mode fiber". IEEE J.Lightwave technology Vol.14. No.10, October 1996

[2] Corning Issue Feb 2001,"Mode Field Diameter Measurements".

[3] Effective Area of Optical Fibers - Definition and Measurement Techniques National Physical Laboratory

[4] Geard Keiser.Optical fiber communication McGraw Hill Int. Editions.

[5] G. J. Foschini and C. D. Poole, "Statistical Theory of Polarization Dispersion in Single-Mode Fibers", Journal of Light wave Technology, vol. LT-9, no. 11, pp 1439-1456, November 1991
[6] J. Sakai, and T. Kimura, "Bending loss of propagation modes in arbitrary-index profile optical fibers", Applied Optics vol. 17, no. 10, pp 1499-1506, May 1978.

[7] J. Sakai, and T. Kimura, "Birefringence Caused by Thermal Stress in Elliptically Deformed Core Optical Fibers", IEEE Journal of Quantum Electronics, vol. QE18, no. 11, pp 1899-1909, November 1982.

[8] S.Ten, M. Edwards's, "An introduction to the fundamentals of PMD in fibers". White paper 2001

[9] Wai, P. K. A., Menyuk. C. R., Polarization mode dispersion, decorrelation, and diffusion in optical fibers with randomly varying birefringence, J. Ligthwave Tech. Jan 2008 